\title{
A seismological analysis of $\delta$ Scuti stars in the Pleiades cluster
}

\author{
L. Fox Machado ${ }^{1}$, F. Pérez Hernández ${ }^{1,2}$, J. C. Suárez ${ }^{3, \star}$, E. Michel ${ }^{4}$, and Y. Lebreton ${ }^{5}$ \\ 1 Instituto de Astrofísica de Canarias, 38205 La Laguna, Tenerife, Spain \\ e-mail: 1 fox@iac.es \\ 2 Departamento de Astrofísica, Universidad de La Laguna, Tenerife, Spain \\ 3 Instituto de Astrofísica de Andalucía (CSIC), 180080 Granada, Spain \\ 4 Observatoire de Paris, LESIA, UMR 8109, 92195 Meudon, France \\ 5 Observatoire de Paris, GEPI, UMR 8111, 92195 Meudon, France
}

Received 7 July 2005 / Accepted 12 September 2005

\begin{abstract}
A comparison between the oscillation frequencies of six multi-periodic $\delta$ Scuti stars of the Pleiades cluster and the eigenfrequencies of rotating stellar models that match the corresponding stellar parameters has been carried out. The assumption that all the stars considered have some common parameters, such as metallicity, distance or age, is imposed as a constraint. As a result, we have a best fit solution associated with a cluster metallicity of $[\mathrm{Fe} / \mathrm{H}] \simeq 0.067$, an age between $70 \times 10^{6}$ and $100 \times 10^{6} \mathrm{yr}$ and a distance modulus of $m_{V}-M_{V}=5.60-5.70 \mathrm{mag}$. All the stars were found to oscillate mainly in non-radial, low degree, low order $p$ modes. Estimates of mass and rotation rates for each star are also obtained.
\end{abstract}

Key words. stars: oscillations - stars: variables: $\delta$ Sct - open cluster and associations: individual: Pleiades

\section{Introduction}

The study of $\delta$ Scuti stars is expected to provide an important diagnostic tool for the structure and evolution of intermediatemass stars at the main sequence evolution and thick-shell hydrogen burning stages. Having masses between 1.5 and $2.5 M_{\odot}$, these pulsating variables develop convective cores during their central hydrogen-burning phases that make them suitable for investigating the hydrodynamic processes occurring in the core.

Although most of the general properties of these stars are well understood, the application of seismological methods to derive their properties has proved to be a difficult task. The problems concerning the modelling of $\delta$ Scuti stars have been discussed in details in recent reviews (e.g. Christensen-Dalsgaard 2000; Kjeldsen 2001). The most acute seem to be the mode selection mechanism and the influence of rotation. Concerning the former, although there is fairly good agreement between the observed frequency range and that derived from instability calculations (e.g. Michel et al. 1999), far more modes than observed are predicted to be unstable. While it is expected that the forthcoming asteroseismology space mission COROT (Baglin et al. 2001) will be able to disentangle the whole spectra of $\delta$ Scuti stars, for the moment the mechanism that could cause an amplitude limitation in some modes is still unknown. As a consequence, mode identification based on the comparison between theoretical and observed frequencies is difficult to attain in general. On the other hand, although sev- eral observational techniques for mode identification have been developed in recent years (Watson 1988; Garrido et al. 1990; Aerts 1996; Kenelly \& Walker 1996; Viskum et al. 1998), in only a few cases have these techniques been successfully applied.

It is well known that most $\delta$ Scuti stars are rapid rotators. This has important effects on the mode frequencies, both directly as a consequence of the changes that must be introduced in the oscillation equations and indirectly through the changes in the equilibrium models. Therefore, successful analysis of rotating $\delta$ Scuti stars requires that rotation should be taken into account consistently at all levels in the analysis.

Despite these problems, in recent years several attempts have been made to interpret the observed spectra of $\delta$ Scuti stars (Goupil et al. 1993; Pérez Hernández et al. 1995; Guzik \& Bradley 1995; Pamyatnykh et al. 1998; Bradley \& Guzik 1996; Hernández et al. 1998; Suárez et al. 2005). Here we address the problem of mode identification for stars in an open cluster. These stars are expected to have a common age and chemical composition. Furthermore, the distance to the cluster is usually known with high accuracy. The constraints imposed by the cluster parameters have proved to be very useful when modelling an ensemble of $\delta$ Scuti stars. The best examples of such studies are found for the variables in the Praesepe cluster (Michel et al. 1999; Hernández et al. 1998). In a similar way, we consider here several $\delta$ Scuti stars of the Pleiades cluster and search for a best fit solution in the sense of a set of stellar

\footnotetext{
* Also associate researcher at Observatoire de Paris.
} 
Table 1. Observational properties of the target stars.

\begin{tabular}{lccccccc}
\hline \hline Star & HD & ST & $m_{V}$ & $B-V$ & $E(B-V)$ & $\begin{array}{c}V \sin i \\
\left(\mathrm{~km} \mathrm{~s}^{-1}\right)\end{array}$ & $\beta$ \\
\hline- & 23628 & A7V & $7.66 \pm 0.03$ & $+0.211 \pm 0.005$ & $0.063 \pm 0.008$ & $150 \pm 15$ & 2.884 \\
V650 Tau & 23643 & A3V & $7.77 \pm 0.02$ & $+0.157 \pm 0.006$ & $0.027 \pm 0.008$ & $175 \pm 18$ & 2.862 \\
- & 23194 & A5V & $8.06 \pm 0.02$ & $+0.202 \pm 0.010$ & $0.076 \pm 0.008$ & $20 \pm 2$ & 2.881 \\
V624 Tau & 23156 & A7V & $8.23 \pm 0.01$ & $+0.250 \pm 0.005$ & $0.046 \pm 0.008$ & $20 \pm 2$ & 2.823 \\
V647 Tau & 23607 & A7V & $8.26 \pm 0.01$ & $+0.255 \pm 0.001$ & $0.057 \pm 0.008$ & $20 \pm 2$ & 2.841 \\
V534 Tau & 23567 & A9V & $8.28 \pm 0.02$ & $+0.360 \pm 0.001$ & $0.084 \pm 0.008$ & $90 \pm 9$ & 2.788 \\
\hline
\end{tabular}

parameters that allows the simultaneous modelling of all the stars considered, and that satisfies all the observables, including the frequencies.

The paper is organized as follows. In Sect. 2, we describe the main characteristics of the Pleiades cluster and the six $\delta$ Scuti stars under study. The modelling, the range of parameters and the calculations of the eigenfrequencies are discussed in Sect. 3. The details of the comparison between observed and theoretical frequencies are discussed in Sect. 4. The results and their discussion are given in Sect. 5. Finally, we present our conclusions in Sect. 6.

\section{The observational material}

The Pleiades (M 45) is a young ( 75-100 Myr), Population I cluster. Because of its proximity, the observational parameters of the Pleiades have been intensively studied. In particular, the metallicity of the cluster is estimated to be between $-0.14 \leq$ $[\mathrm{Fe} / \mathrm{H}] \leq+0.13$ (e.g. $-0.034 \pm 0.024$ Boesgaard \& Friel 1990, $0.0260 \pm 0.103$ Cayrel de Strobel 1990, $-0.11 \pm 0.03$ Grenon 2001).

Until recently, there was a dispute regarding the distance of the Pleiades cluster from the Earth. While the determination of the cluster distance from direct parallax measurements of HIPPARCOS gives $116.3 \pm_{3.2}^{3.3} \mathrm{pc}\left(m_{V}-M_{V}=5.33 \pm 0.06 \mathrm{mag}\right.$, Mermilliod et al. 1997), the previously accepted distance, which is based on comparing the cluster's main sequence with that of nearby stars, was $\approx 130$ pc corresponding to a distance modulus of about $5.60 \mathrm{mag}$ (e.g. $5.65 \pm 0.38 \mathrm{mag}$, Eggen 1986; $5.60 \pm 0.05 \mathrm{mag}$, Pinsonneault et al. 1998; $5.61 \pm 0.03 \mathrm{mag}$, Stello \& Nissen 2001). As will be discussed in Sect. 5, the distance derived in the research presented here agrees with the latter value.

To date, six $\delta$ Scuti stars are known in the Pleiades. In a survey of variability in the cluster, Breger (1972) found $\delta$ Scuti type oscillations in four stars. The remaining two were recently reported to be $\delta$ Scuti stars by Koen (1999) and Li et al. (2002). Some observational properties of these stars are given in Table 1. The projected rotational velocities, $v \sin i$, were obtained from Morse et al. (1991) and Uesugi \& Fukuda (1982). A $10 \%$ uncertainty in these quantities has been assumed. The spectral types (ST) and $\beta$ parameters were obtained from the SIMBAD database in Strasbourg (France). The errors in the photometric parameters $(B-V)$ and $m_{V}$ are estimated from the dispersion between different measurements of these quantities. The errors in reddening are taken from Breger (1986). We shall use these uncertainties in Sect. 3.2.
Table 2. Observed frequencies used in this work. The data were obtained from different STEPHI campaigns as detailed in the text.

\begin{tabular}{lc|ll}
\hline \hline Star & $\begin{array}{c}v \\
(\mu \mathrm{Hz})\end{array}$ & Star & $\begin{array}{c}v \\
(\mu \mathrm{Hz})\end{array}$ \\
\hline HD 23628 & 191.8 & V624 Tau & 242.9 \\
& 201.7 & & 409.0 \\
& 376.6 & & 413.5 \\
\cline { 1 - 1 } V650 Tau & 197.2 & & 416.4 \\
& 292.7 & & 451.7 \\
& 333.1 & & 489.4 \\
& 377.8 & & 529.1 \\
\hline HD 23194 & 533.6 & V647 Tau & 236.6 \\
& 574.9 & & 304.7 \\
\cline { 1 - 1 } V534 Tau & 234.2 & & 315.6 \\
& 252.9 & & 374.4 \\
& 307.6 & & 405.8 \\
& 377.9 & & 444.1 \\
& 379.0 & & \\
& 448.1 & & \\
& 525.0 & & \\
\hline
\end{tabular}

In recent years, the $\delta$ Scuti stars of the Pleiades cluster have been observed in several campaigns of the STEPHI multisite network (Michel et al. 2000). The information on the oscillation frequencies of the stars used here has been obtained from those campaigns and are published elsewhere (Belmonte \& Michel 1991; Michel et al. 1991; Liu et al. 1999, 2002; Li et al. 2004; Fox-Machado et al. 2002). The frequency peaks detected with a confidence level above $99 \%$ are summarized in Table 2. We note that the frequency resolution in a typical STEPHI campaign (three weeks) is $\Delta v \sim 0.5 \mu \mathrm{Hz}$.

Figure 1 illustrates a colour-magnitude diagram of the Pleiades cluster in the region where the target stars are located. The filled circles corresponds to the $\delta$ Scuti stars. The apparent magnitudes, $V$, colours, $(B-V)$ and membership used are contained in the WEBDA database (Mermilliod 2000). The Pleiades shows differential reddening with significant excess in the southwest with an average value of $E(B-V) \approx 0.04$ (e.g. van Leeuwen 1999; Breger 1986; Hansen-Ruiz \& van Leeuwen 1997; Pinsonneault et al. 1998). In particular, we adopted the reddening for individual stars as given by Breger (1986) if present, otherwise the average of $E(B-V)=0.04$ was used. Stars known to be spectroscopic binaries were rejected.

Given the young age of the Pleiades cluster, we expect all the $\delta$-Scuti stars to be at an early evolutionary stage on the main sequence. From the figure it follows that while V647 Tau and V624 Tau have similar masses, V650 Tau, HD 23194 and 


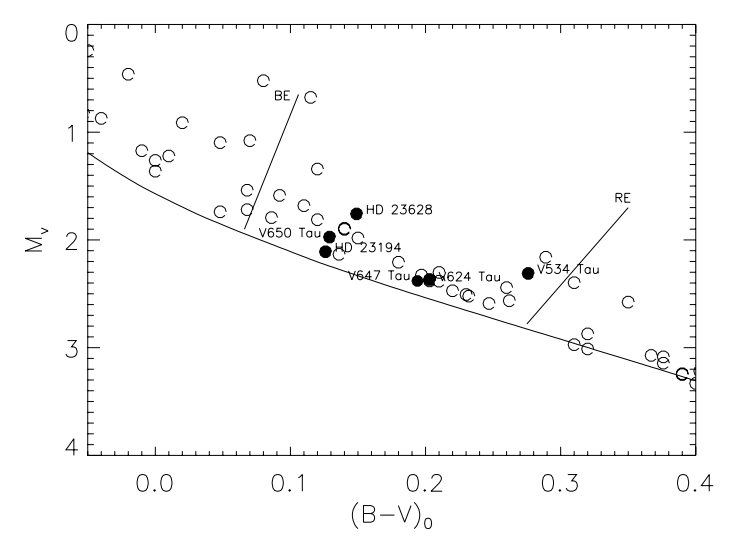

Fig. 1. HR diagram of the Pleiades cluster. Only the region around the instability strip is shown. The target stars are represented with filled circles. The blue and red edges of the instability strip are indicated by continuous lines and were taken from Rodríguez et al. (2000). An isochrone of $70 \mathrm{Myr}$ is also shown.

HD 23628 are slightly more massive. V534 Tau, located near the red edge of the instability strip, is the coolest star in the ensemble.

\section{The modelling}

In this section we shall explain how we have computed the stellar models, the corresponding eigenfrequencies and how we have constrained the stellar parameters used in the forward comparison with the observed frequencies.

\subsection{The stellar models}

We have considered stellar models with input physics appropriate to the mass range covered by $\delta$ Scuti stars. In particular, the stellar models were computed using the CESAM evolutionary code (Morel 1997). The nuclear reaction rates are from Caughlan \& Fowler (1988), the equation of state is from Eggleton et al. (1973), the opacities from the OPAL project (Iglesias \& Roger 1996) complemented at low temperatures by Kurucz data (1991), and the atmosphere is computed using the Hopf's $T(\tau)$ law (Mihalas 1978). The convection is described according to the classical mixing-length theory (MLT hereafter). In particular, we have considered an MLT parameter of $\alpha_{\mathrm{MLT}}=l / H_{\mathrm{p}}=1.52$, where $H_{\mathrm{p}}$ is the pressure scaleheight. We have considered a fixed value for $\alpha_{\mathrm{MLT}}$ because this parameter is not expected to have any significant influence on the global structure of the evolution models considered for our target stars.

We have considered different sets of initial chemical compositions in order to cover the Pleiades $[\mathrm{Fe} / \mathrm{H}]$ observed range (see Sect. 2) and different initial He abundances. The values used are given in Table 3. For each initial chemical composition we have computed models with and without core overshooting, $\alpha_{\mathrm{ov}}=0.20$ in the former case (Schaller et al. 1992).

Although the final comparison with observations is done considering rotating models, our procedure requires the computation of non-rotating models and the corresponding isochrones. Hence, sequences of non-rotating models were
Table 3. Metallicity of the models. $[\mathrm{Fe} / \mathrm{H}]=\log (Z / X)_{*}-\log (Z / X)_{\odot}$, with $(Z / X)_{\odot}=0.0245$ from Grevesse \& Noels (1993).

\begin{tabular}{cccrc}
\hline \hline$X_{0}$ & $Y_{0}$ & $Z_{0}$ & {$[\mathrm{Fe} / \mathrm{H}]$} & $\alpha_{\mathrm{ov}}$ \\
\hline 0.735 & 0.250 & 0.015 & -0.0794 & $0.0-0.2$ \\
0.685 & 0.300 & 0.015 & -0.0488 & $0.0-0.2$ \\
0.700 & 0.280 & 0.020 & 0.0668 & $0.0-0.2$ \\
0.725 & 0.250 & 0.025 & 0.1484 & $0.0-0.2$ \\
0.675 & 0.300 & 0.025 & 0.1794 & $0.0-0.2$ \\
\hline
\end{tabular}

calculated for masses between $0.8 M_{\odot}$ and $5.0 M_{\odot}$, from the ZAMS to the sub-giant branch and the corresponding isochrones computed for each sets of parameters in Table 3. In the following analysis we shall consider three ages, $A$, of $70 \mathrm{Myr}, 100 \mathrm{Myr}$ and $130 \mathrm{Myr}$. Finally, for comparison with the observations three distance moduli, $d$, of 5.50, 5.60 and 5.70 mag were considered except for models with $[\mathrm{Fe} / \mathrm{H}]=$ -0.0488 , in which case an additional value of $d=5.39$ mag was used.

Figure 1 shows an example of such isochrones computed with the following parameters: $[\mathrm{Fe} / \mathrm{H}]=0.0668, \alpha_{\mathrm{ov}}=0.2$, $A=70 \mathrm{Myr}$ and $d=5.70 \mathrm{mag}$. The isochrones were calibrated from $\left[T_{\text {eff }}, \log \left(L / L_{\odot}\right)\right]$ to $\left(B-V, M_{V}\right)$ by using the Schmidt-Kaler (1982) calibration for magnitudes and the relationship between $T_{\text {eff }}$ and $B-V$ of Sekiguchi \& Fukugita (2000) for the colours. As can be seen in Fig. 1, in the case illustrated here the isochrone matches the observed colour-magnitude diagram. For some combinations of high metallicity and low distance modulus the isochrone fit is not satisfactory but we have not rejected those combinations to allow for an independent determination of the cluster parameters from the oscillation frequencies. Hence we have considered the entire combination of cluster parameters given in Table 3 and the three ages given above.

\subsection{Photometric effect of rotation on the HR diagram}

It is known that fast rotation affects the position of a star in the colour-magnitude diagram (e.g. Tassoul 1978). In particular a ZAMS rotating model has a smaller luminosity and mean $T_{\text {eff }}$ than a non-rotating model with the same mass and chemical composition. Also, the magnitude and the colour of a rotating star depend on the aspect angle, $i$, between the line of sight and the rotation axis. In particular, a rotating star seen pole-on appears brighter and hotter than the same star seen equator-on.

We now search for the range of masses and angular rotations suitable for each star. To do this we shall use the results of Pérez Hernández et al. (1999), in which the correction to the photometric magnitudes of non-rotating models needed to obtain those of rotating models were computed for main sequence stars of spectral type A0 to F5. In this calculation, we shall take into account the observed $v \sin i$ for each star. As an example, Fig. 2 illustrates the corrections due to rotation in the particular case of the $\delta$ Scuti type star V650 Tau with $v \sin i=175 \mathrm{~km} \mathrm{~s}^{-1}$. The actual position of the star is shown with a thick dot upon which there is a cross that gives the errors in the estimation of 


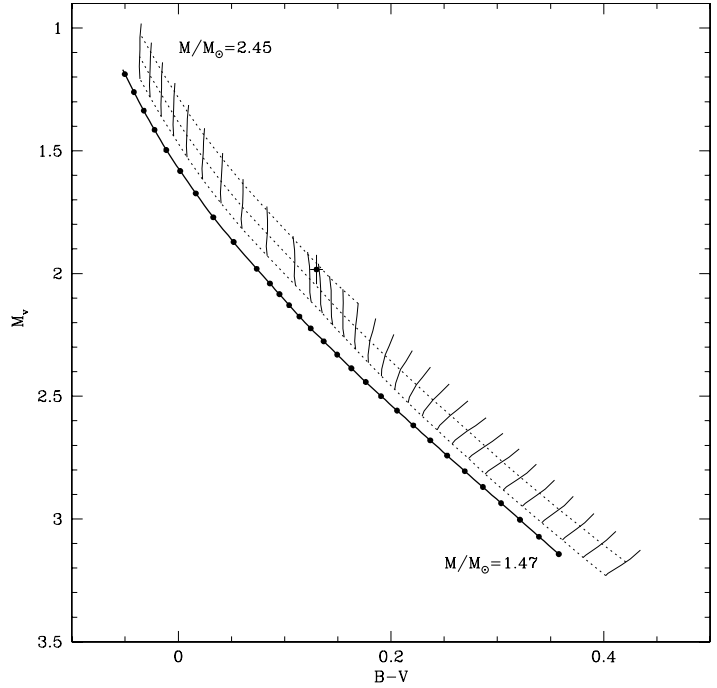

Fig. 2. Colour-magnitude diagram illustrating the photometric corrections due to rotation in the case of V650 Tau $\left(v \sin i=175 \mathrm{~km} \mathrm{~s}^{-1}\right)$. The thick continuous line is the same isochrone represented in Fig. 1 $\left([\mathrm{Fe} / \mathrm{H}]=0.0668, \alpha_{\mathrm{ov}}=0.2, A=70 \mathrm{Myr}, d=5.7 \mathrm{mag}\right)$. Some models are shown with filled circles upon the isochrone. The masses of the first and last models are indicated. The small tracks represent the photometric corrections due to rotation for each the models indicated on the isochrone when $i$ runs from $i=90^{\circ}$ to $i=i_{\min }$. The corrections at fixed $i$ are represented by dotted lines.

$\left(M_{V}\right)_{0}$ and $(B-V)_{0}$ calculated according the following expressions:

$\sigma\left[(B-V)_{0}\right]=\sqrt{\sigma^{2}(B-V)+\sigma^{2}[E(B-V)]}$,

$\sigma\left[\left(M_{V}\right)_{0}\right]=\sqrt{\sigma^{2}(d)+\sigma^{2}\left(m_{V}\right)+(3.2 \sigma[E(B-V)])^{2}}$,

where $\sigma(d)=0.06$ is the error in the distance modulus. The errors in the magnitude $M_{V}$, colour index $(B-V)$ and reddening $E(B-V)$ are given in Table 1 .

The range of masses and rotational velocities of the star depends on the stellar parameters considered and hence needs to be computed for each combination in Table 3. In particular, in the example illustrated in Fig. 2, the same cluster parameters as in Fig. 1 were considered $\left([\mathrm{Fe} / \mathrm{H}]=0.0668, \alpha_{\text {ov }}=0.2\right.$, $A=70 \mathrm{Myr}$ and $d=5.70 \mathrm{mag}$ ). The isochrone is represented by a thick continuous line and some of its models are shown by dots. For each model on the isochrone there is a small track corresponding to the photometric corrections due to rotation computed by using the projected velocity $v \sin i$ of the star and changing the angle of inclination from $i=90^{\circ}$ to the minimum angle $i=i_{\text {min }}$ corresponding to $\sim 0.90 \Omega_{\mathrm{br}}$, where $\Omega_{\mathrm{br}}$ is the break-up angular rotational velocity. This angular velocity is given by the balance between the gravitational attraction and the centrifugal force at the equator. The dotted lines give the corrections at fixed angles: $i=90^{\circ}$ (the closest line to the isochrone), $i=50^{\circ}$ (intermediate line) and $i=39^{\circ}$ (the reddest line). It is evident that the actual position of the star, including the error box, is associated to a domain of non-rotating models. In this case we have obtained for the star considered a range of masses of $[1.86,1.91] M_{\odot}$ and a range of angles of inclination of $\left[39^{\circ}, 50^{\circ}\right]$. Nonetheless, in order to take into account

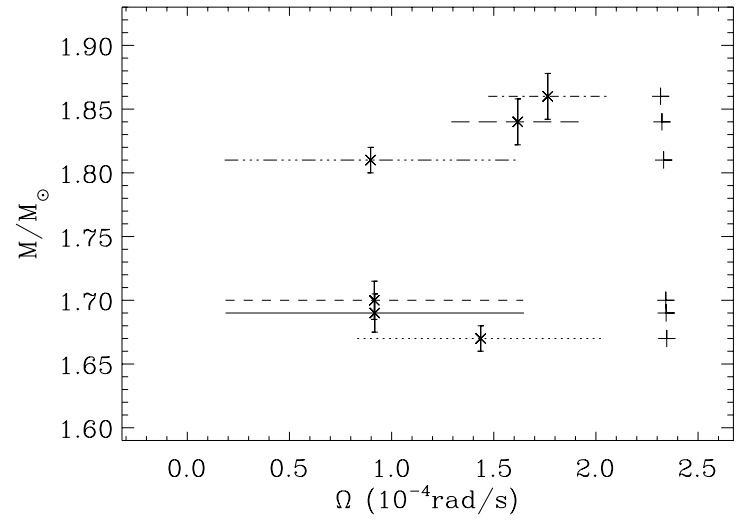

Fig. 3. Range of rotation rates against masses estimated for the six stars from the photometric corrections due to rotation applied to the same isochrone depicted in Fig. 2. The vertical error bars give the estimated range of mass for each star. The crosses give the values of the break-up angular rotational velocity, $\Omega_{\mathrm{br}}$. The ranges of $\Omega$ and $M$ are associated with stars as follows: dot-dashed line for V650 Tau $\left(v \sin i=175 \mathrm{~km} \mathrm{~s}^{-1}\right)$; long-dashed line for HD 23628 $\left(v \sin i=150 \mathrm{~km} \mathrm{~s}^{-1}\right)$; three-dots dashed line for HD $23194(v \sin i=$ $\left.20 \mathrm{~km} \mathrm{~s}^{-1}\right)$; dashed-line for V647 Tau $\left(v \sin i=20 \mathrm{~km} \mathrm{~s}^{-1}\right)$; continuous line for V624 Tau $\left(v \sin i=20 \mathrm{~km} \mathrm{~s}^{-1}\right)$ and dotted line for V534Tau $\left(v \sin i=90 \mathrm{~km} \mathrm{~s}^{-1}\right)$.

possible errors coming from the isochrone calibration we shall consider a wider range of aspect angles $i=\left[i_{\min }, 90^{\circ}\right]$. The same procedure is carried out for the other stars. Also one needs to consider all the metallicities, ages, overshooting parameters and distance moduli for the whole set of stars.

Having obtained ranges of $M$ and values of $i_{\min }$, we proceed to find the corresponding range of angular rotational velocities $\Omega$, for which an estimate of the equatorial radius, $R_{\text {eq }}$, of the rotating model is needed. Under the assumption of uniform rotation and approximating the surface of the star by a Roche surface (see e.g. Pérez Hernández et al. 1999) one has:

$R_{\mathrm{eq}} \simeq R_{0} \frac{3}{\omega} \cos \left\{\frac{1}{3}[\pi+\operatorname{arcos} \omega]\right\}$,

where $\omega \simeq \Omega / \Omega_{\mathrm{br}}, \Omega_{\mathrm{bq}}^{2} \simeq 8 G M /\left(27 R_{0}^{3}\right)$ and $R_{0}$ is the polar radius. As noted in Pérez Hernández et al. (1999), the polar radius can be approximated by the radius of a spherical non-rotating model with the same mass and evolutionary state (since our stars are close to the ZAMS, a non-rotating model of the same mass and age is suitable for this error-box estimation).

Since on the other hand the angular rotation is related to the equatorial radius by

$\Omega \simeq \frac{(v \sin i)_{\mathrm{obs}}}{R_{\mathrm{eq}} \sin i}$,

it is possible to carry out a simple iterative process to obtain a range of possible rotations, $\left[\Omega_{\min }, \Omega_{\max }\right]$, from the previously obtained range of angle of inclinations $\left[i_{\min }, 90^{\circ}\right]$ and masses. Since for given data parameters, the mass of the star is obtained with an uncertainty of $\sim 0.02 M_{\odot}$ this iterative process was carried out for just one mass.

As an example, Fig. 3 shows the ranges of $\Omega$ obtained for the six stars as a function of the mass of the models considering the same cluster parameters as in Fig. 2. Lines of different 
types give the estimates for the different stars as indicated in the figure. A similar procedure needs to be considered for all sets of parameters in Table 3.

With the information obtained above we compute evolutionary sequences of rotating models with the same input physics as the non-rotating models but with appropriate initial angular rotational velocities in order to match as closely as possible the extreme values in the interval $\left[\Omega_{\min }, \Omega_{\max }\right]$ at the final age. Solid-body rotation and conservation of global angular momentum during the evolution were assumed during the calculus. In order to have a better overview of the rotation rates of the stars we have also computed models with an intermediate value of $\Omega_{\text {mid }} \approx 0.5 \Omega_{\mathrm{br}}$. A total amount of 1620 sequences of rotating models were finally obtained for the whole ensemble of stars.

\subsection{Theoretical oscillation frequencies}

The eigenfrequencies of the rotating models previously described have been calculated using the oscillation code FILOU (Tran Minh et al. 1996; Suárez 2002). We have considered frequency perturbations up to second order in the rotation rate. Frequencies of the modes are labelled in the usual way: $n, l, m$ for the radial order, degree and azimuthal order. The radial orders of a given mode are assigned according to the Scuflaire criterion (1974) with $n>0$ for the $p$ modes, $n<0$ for the $g$ modes, $n=1$ for the fundamental radial mode of degree 0 and 1 , and $n=0$ for the fundamental mode with the $l=2$. Eigenfrequencies were computed up to $l=2$. For geometrical reasons higher degree modes are expected to have considerably smaller amplitudes. The theoretical frequencies cover the frequency range of the observed pulsation peaks (see Table 2). Coupling between modes is not considered in the present work.

The estimated interval of $\Omega_{\mathrm{rot}}$ for all the stars may be as large as $\Delta \Omega_{\text {rot }} \sim 1.6 \times 10^{-4} \mathrm{rad} / \mathrm{s}$ which in terms of cyclic rotational frequency corresponds to $\Delta v_{\text {rot }} \sim 25 \mu \mathrm{Hz}$ (see for instance Fig. 3). After some tests, we found that a satisfactory comparison between the observed and theoretical frequencies cannot be achieved by using only the frequencies computed for models with three $\Omega_{\text {rot }}$ within this interval. To overcome this difficulty once we had the mode frequencies for each series of models (three $\Omega_{\text {rot }}$ for fixed $M,[\mathrm{Fe} / \mathrm{H}], \alpha_{\mathrm{MLT}}, \alpha_{\mathrm{ov}}, d$ and $A$ ), we proceeded to interpolate the results on $\Omega_{\text {rot }}$ in 21 equally spaced points covering the interval $\left[\Omega_{\min }, \Omega_{\max }\right]$. A quadratic spline interpolation was applied. In order to evaluate the goodness of the interpolation we also computed the eigenfrequencies for several randomly selected models. We have found that there is a good agreement between the interpolated and theoretical frequencies up to $v \sim 600 \mu \mathrm{Hz}(n \sim 6)$, the average of absolute separation being $\left|v_{\text {int }}-v_{\text {cal }}\right| \sim 0.3 \mu \mathrm{Hz}$. The disagreement found beyond this value can be explained by the fact that the second order effect of rotation is enhanced at higher frequencies. In any case, as can be seen in Table 2, the highest frequency we are dealing with is $\simeq 574 \mu \mathrm{Hz}$.
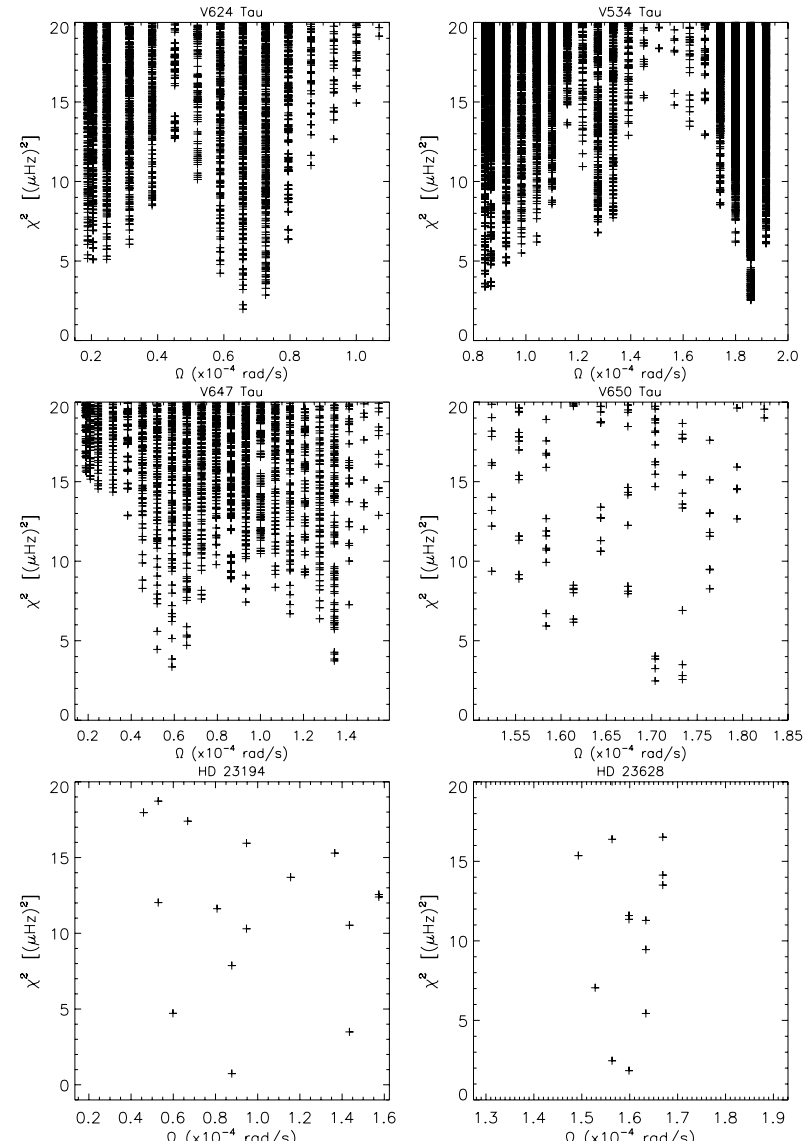

Fig. 4. $\chi^{2}$ against $\Omega$ for six models with common parameters $[\mathrm{Fe} / \mathrm{H}]$, $\alpha_{\mathrm{ov}}, d$ and $A$.

\section{Method of comparison}

Once we have the mode frequencies for all sets of parameters we compare the observational frequencies $\left(v_{\mathrm{obs}}\right)$ with the theoretical ones $\left(v_{\text {cal }}\right)$ at each interpolated $\Omega_{\text {rot }}$.

Let us consider a rotating model with given parameters for just one star. Then, for all the possible combinations between the observed and computed frequencies, we compute the quantity $\chi^{2}$ given by

$\chi^{2}=\frac{1}{N} \sum_{j=1}^{N}\left(v_{\mathrm{obs}, j}-v_{\mathrm{cal}, j}\right)^{2}$,

where $N$ is the number of observational frequencies. In this computation it is understood that each theoretical frequency is assigned to one observed frequency at most.

As a first step, in order fully to exploit the collective behaviour of stars within open cluster we consider the solutions of the six stars with given cluster parameters of $[\mathrm{Fe} / \mathrm{H}], \alpha_{\mathrm{ov}}, d$ and $A$. The models corresponding to each star differ in the mass and the rotation rate. As an example, Fig. 4 shows $\chi^{2}$ against angular rotational velocity, $\Omega$, for the six models corresponding to the same parameters as in Fig. 2 but without overshooting $\left([\mathrm{Fe} / \mathrm{H}]=0.0668, \alpha_{\mathrm{ov}}=0.0, d=5.70 \mathrm{mag}\right.$ and $\left.A=70 \mathrm{Myr}\right)$. Each panel corresponds to the star indicated in the figure. For clarity, only solutions with $\chi^{2}<20\left(\mu \mathrm{Hz}^{2}\right)$ are shown. Similar 


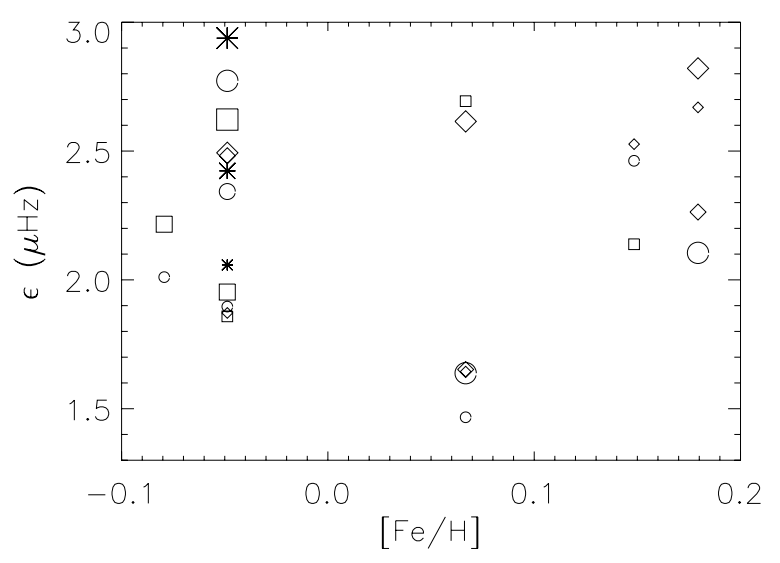

Fig. 5. Minimum values of the mean square root of the difference between observed and theoretical frequencies for models with the same values of $[\mathrm{Fe} / \mathrm{H}], \alpha_{\mathrm{ov}}, d$ and $A$ against $[\mathrm{Fe} / \mathrm{H}]$. For clarity, only models without overshooting are shown. The symbols are related to different values of $A$ and $d$ as follows: asterisks ( $d=5.39 \mathrm{mag}$ ), squares ( $d=5.50 \mathrm{mag})$, diamonds $(d=5.60 \mathrm{mag})$, circles $(d=5.70 \mathrm{mag})$, small (70 Myr), middle (100 Myr) and big (130 Myr).

features are found for other metallicity, distance moduli and ages, but the lowest $\chi^{2}$ may appear with rather high values.

Since we expect the same $[\mathrm{Fe} / \mathrm{H}], d$ and $A$ for all the stars in the cluster we shall assume that the best fits should happen simultaneously in the six stars despite differences in rotation rate and masses of the models. Hence we proceed to calculate the mean square root of the lowest $\chi^{2}$ found in each model for given common parameters by means of the following expression:

$\epsilon=\sqrt{\frac{1}{6} \sum_{i=1}^{6}\left(\chi_{\min , i}\right)^{2}}$

Figure 5 shows $\epsilon$ against $[\mathrm{Fe} / \mathrm{H}]$ for models without overshooting. For the sake of clarity only the points with $\epsilon \leq 3.0 \mu \mathrm{Hz}$ are shown. It can be seen that the solution with the smallest $\epsilon$ corresponds to $[\mathrm{Fe} / \mathrm{H}]=0.0668$. A plot of similar characteristics is obtained for models with overshooting.

Since only those identifications obtained from Eq. (5) with low $\chi^{2}$ values are of interest, we require that for each star the solution must have $\chi \leq 3.5 \mu \mathrm{Hz}$. In Fig. 5 we reject those solutions that have at least one model with $\chi>3.5 \mu \mathrm{Hz}$ for all the parameters and combinations between observed and theoretical frequencies. With this restriction we found that only those solutions associated with the models represented by the four lowest points in Fig. 5 should be considered. Similar solutions were found for models with overshooting. All these models are listed in Table 4 and will be analysed in detail later.

We shall use a geometrical argument to reduce further the number of solutions so far available for each star. To this end, we take into account that the visibility of each mode depends on the angle of inclination. Following Pesnell (1985), we illustrate in Fig. 6 the spatial response function $S_{l m}$ against $i$, for mode degree $l=0,1,2$. For simplicity, limb-darkening has been neglected. It can be seen that for $i \approx 90^{\circ}$ only even $l+m$ values can be detected, while for $i \approx 0^{\circ}$ only modes with $m=0$ will be visible.
Table 4. Ensembles of models with and without overshooting, which, after applying a threshold of $\chi \leq 3.5 \mu \mathrm{Hz}$, have potential valid solution (see text for details).

\begin{tabular}{cccccc}
\hline \hline \multicolumn{5}{c}{$[\mathrm{Fe} / \mathrm{H}]=0.0668$} \\
\hline & $\begin{array}{c}d \\
\alpha_{\mathrm{ov}}=0.00\end{array}$ & $\begin{array}{c}c \\
\alpha_{\mathrm{ov}}=0.20 \\
(\mathrm{mag})\end{array}$ & & $\begin{array}{c}d \\
(\mathrm{Myr})\end{array}$ & $\begin{array}{c}A \\
(\mathrm{Myr})\end{array}$ \\
\hline $\mathrm{C} 1$ & 5.70 & 70 & $\mathrm{D} 1$ & 5.70 & 70 \\
$\mathrm{C} 2$ & 5.70 & 130 & $\mathrm{D} 2$ & 5.60 & 100 \\
$\mathrm{C} 3$ & 5.60 & 70 & $\mathrm{D} 3$ & 5.60 & 130 \\
$\mathrm{C} 4$ & 5.60 & 100 & $\mathrm{D} 4$ & 5.70 & 100 \\
\hline
\end{tabular}

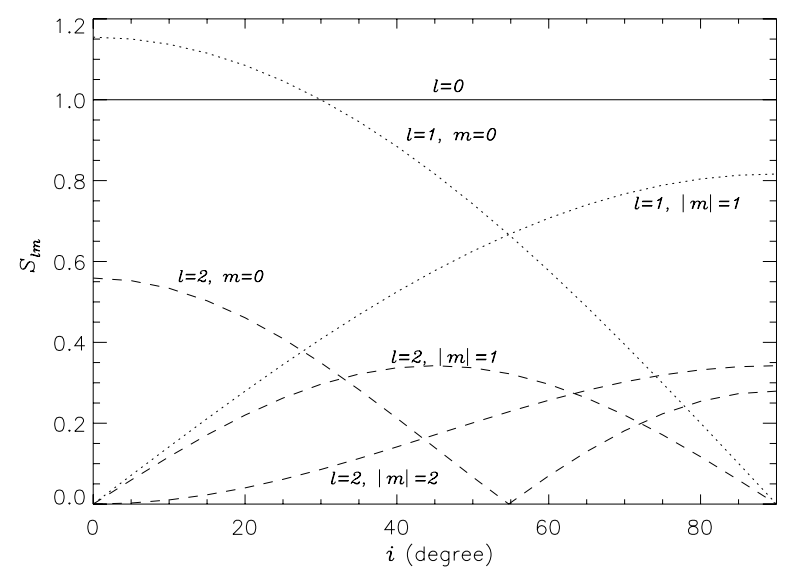

Fig. 6. Variation of visibility amplitude with the inclination angle for radial $l=0$ (continuous line) and non-radial $l=1$ (dotted lines) and $l=2$ (dashed lines) modes.

We introduce an hypothesis based on the visibility of the modes: we reject any solution with one or more modes with $S_{l m}^{\prime}<0.1$. After applying this constraint we were able to limit the analysis to four sets in Table 4, two with $\alpha_{\mathrm{ov}}=0.0$ : C1 $(d=5.70, A=70), \mathrm{C} 3(d=5.60, A=70)$ and two with $\alpha_{\text {ov }}=0.2: \mathrm{D} 1(d=5.70, A=70), \mathrm{D} 2(d=5.60, A=100)$. We note that while for the stars HD 23628 and HD 23194 only a few identifications $(<8)$ remain, the number of identifications for V624 Tau, V534 Tau, V647 Tau and V650 Tau remained larger than 100 in most cases.

\section{Results and discussion}

In order to discuss the results we shall introduce a parameter $\Delta$ associated with each star in each identification and given by

$\Delta=\max \left(\left|v_{\mathrm{obs}}-v_{\mathrm{cal}}\right|\right)$.

Table 5 lists the number of solutions for the six stars in each ensemble. Different maximum values of $\Delta$ have been considered. Certain features can be derived from these general results:

- The resulting masses for each star are similar for all the solutions. 
Table 5. Number of possible solutions for models in each ensemble for different values of $\Delta$ (in $\mu \mathrm{Hz}$ ).

\begin{tabular}{|c|c|c|c|c|c|c|c|c|c|c|}
\hline & \multicolumn{10}{|c|}{$[\mathrm{Fe} / \mathrm{H}]=0.0668$} \\
\hline & \multicolumn{5}{|c|}{$\alpha_{\mathrm{ov}}=0.00$} & \multicolumn{5}{|c|}{$\alpha_{\mathrm{ov}}=0.20$} \\
\hline & $\Delta(\mu \mathrm{Hz}) \leq$ & 1.0 & 2.0 & 3.0 & 3.5 & $\Delta(\mu \mathrm{Hz}) \leq$ & 1.0 & 2.0 & 3.0 & 3.5 \\
\hline & $\frac{M}{M_{\odot}}$ & & & & & $\frac{M}{M_{\odot}}$ & & & & \\
\hline & $\mathrm{C} 1$ & & & & & D1 & & & & \\
\hline V624 Tau & 1.70 & 0 & 0 & 12 & 18 & 1.69 & 0 & 0 & 0 & 12 \\
\hline V534 Tau & 1.67 & 0 & 0 & 0 & 16 & 1.67 & 0 & 0 & 0 & 0 \\
\hline V647 Tau & 1.70 & 0 & 0 & 0 & 2 & 1.70 & 0 & 0 & 0 & 2 \\
\hline V650 Tau & 1.86 & 0 & 0 & 7 & 13 & 1.86 & 0 & 2 & 4 & 4 \\
\hline HD 23194 & 1.80 & 0 & 1 & 2 & 3 & 1.81 & 0 & 0 & 3 & 3 \\
\hline HD 23668 & 1.84 & 0 & 1 & 2 & 4 & 1.84 & 0 & 0 & 2 & 3 \\
\hline & $\mathrm{C} 3$ & & & & & D2 & & & & \\
\hline V624 Tau & 1.68 & 0 & 0 & 6 & 12 & 1.68 & 0 & 0 & 0 & 0 \\
\hline V534 Tau & 1.66 & 0 & 0 & 0 & 24 & 1.66 & 0 & 0 & 0 & 0 \\
\hline V647 Tau & 1.69 & 0 & 0 & 0 & 2 & 1.69 & 0 & 0 & 0 & 3 \\
\hline V650 Tau & 1.86 & 0 & 0 & 0 & 0 & 1.86 & 0 & 0 & 0 & 3 \\
\hline HD 23194 & 1.79 & 1 & 1 & 2 & 2 & 1.79 & 1 & 1 & 1 & 2 \\
\hline HD 23668 & 1.82 & 0 & 0 & 3 & 4 & 1.84 & 0 & 2 & 3 & 6 \\
\hline
\end{tabular}

- The number of solutions for a given star at fixed $\Delta$ associated with models with overshooting is smaller than without it.

- There are no solutions with $\Delta \leq 3.0 \mu \mathrm{Hz}$ for the stars V534 Tau and V647 Tau, while the star HD 23194 shows solutions even with $\Delta \leq 1.0 \mu \mathrm{Hz}$.

- In order to find a set of models that has at least one solution simultaneously for all the stars a value of $\Delta \simeq 3.5 \mu \mathrm{Hz}$ is needed. This solution is found for $\mathrm{C} 1$ that corresponds to the cluster parameters $[\mathrm{Fe} / \mathrm{H}]=0.0668, \alpha_{\mathrm{ov}}=0.0, d=$ 5.70 and $A=70$, and it coincides with the minimum in Fig. 5.

- The remaining ensembles in Table 5 have at least one solution simultaneously when the value of $\Delta$ is increased slightly. In particular, for $\mathrm{C} 3$ a value of $\Delta \simeq 4.5 \mu \mathrm{Hz}$ is needed to obtain a solution while for D1 and D2 a value of $\Delta \simeq 3.8 \mu \mathrm{Hz}$ is required.

We have found that the identifications and the range of aspect angles derived for each star are similar for all the solutions. This agreement may be due to the fact that the estimated range of mass and radius, and hence of mean densities, is similar for all the solutions. At the ages considered the overshooting has a negligible effect on the models.

Table 6 summarizes the parameters estimated for the cluster as well as for each star associated with the possible identifications. The radial orders of these modes are consistent with growth rate predictions (Suárez et al. 2002). While these identifications cannot be considered as definitive but rather as "best fit solutions", some information on the stars could be derived. On the one hand, those stars with smaller masses (V624 Tau, V534 Tau and V647 Tau) have more than six frequencies and could have simultaneously excited radial and non-radial oscillations although at most two radial modes. Following Hernández et al. (1998), at most two radial modes were found to be excited for the $\delta$ Scuti stars in the Praesepe cluster. On the other hand, the most massive stars, V650 Tau, HD 23628 and HD 23194, with a mean mass of $1.83 M_{\odot}$ present on average few observational frequencies $(N \leq 4)$ that might be associated only with non-radial modes. With the present observational data it remains unclear, however, whether this is a general trend for $\delta$ Scuti stars.

A comparison of observed and computed frequencies is shown in Fig. 7. In each panel the solid lines represent the observational frequencies with their corresponding amplitudes. The "best fit" theoretical frequencies are represented by dotted lines. The stars are arranged from top to bottom according to their magnitude.

From this figure it follows that there is an asymmetric triplet in the observational spectrum of V624 Tau. We have found that for all the solutions the identification is always a ( $l=1, n=3$ ) mode split by rotation. In turn, this implies that an independent estimate of the mean rotation rate can be obtained from the rotational splitting, up to second order, given by

$\frac{v_{-m}-v_{m}}{2 m} \sim \frac{\Omega_{\mathrm{rot}}}{2 \pi}\left(1-C_{n l}\right), \quad m=1,2, \ldots, l$.

We have found complete agreement between the cyclic rotational frequency computed from the above expression and those derived from the mode identification, the differences being $\sim 0.03-0.04 \mu \mathrm{Hz}$. We have also found a fairly good agreement between the observations and the models in the differences $v_{-m}-v_{0}$ and $v_{+m}-v_{0}$.

If the estimated inclination angles for the stars are correct, all of them could be rapid rotators $\left(v_{\text {rot }}>10 \mu \mathrm{Hz}\right)$ except for 
Table 6. Summary of parameters derived for the Pleiades cluster as well as for $\delta$ Scuti stars with the possible identification of the frequency modes.

\begin{tabular}{|c|c|c|c|c|c|}
\hline \multicolumn{6}{|c|}{$[\mathrm{Fe} / \mathrm{H}]=0.0668, \alpha_{\mathrm{ov}}=[0.00-0.20], m_{V}-M_{V}=[5.60-5.70], A=[70-100] \times 10^{6}$ years } \\
\hline Star & $\begin{array}{c}v \\
(\mu \mathrm{Hz})\end{array}$ & $\begin{array}{l}\text { Identification } \\
\quad(n, l, m)\end{array}$ & Star & $\begin{array}{c}v \\
(\mu \mathrm{Hz})\end{array}$ & $\begin{array}{l}\text { Identification } \\
\quad(n, l, m)\end{array}$ \\
\hline $\begin{array}{l}\text { V624 Tau } \\
M=[1.68-1.72] M_{\odot} \\
v_{\text {rot }}=[3-5] \mu \mathrm{Hz} \\
i=\left[37^{\circ}-67^{\circ}\right]\end{array}$ & $\begin{array}{l}242.9 \\
409.0 \\
413.5 \\
416.4 \\
451.7 \\
489.4 \\
529.1\end{array}$ & $\begin{array}{c}(1,0,0) \\
(3,1,1) \\
(3,1,0) \\
(3,1,-1) \\
(3,2,-2),(4,0,0) \\
(4,1,0),(4,1,1) \\
(4,2,-1),(4,2,-2) \\
(5,0,0)\end{array}$ & $\begin{array}{l}\text { V650 Tau } \\
M=[1.84-1.88] M_{\odot} \\
v_{\text {rot }}=[25-28] \mu \mathrm{Hz} \\
i=\left[60^{\circ}-70^{\circ}\right]\end{array}$ & $\begin{array}{l}197.2 \\
292.7 \\
333.1 \\
377.8\end{array}$ & $\begin{array}{c}(1,1,1) \\
(0,2,-2),(2,2,2) \\
(3,1,1),(3,2,2) \\
(2,2,-2),(3,1,0) \\
(3,1,-1),(3,2,1)\end{array}$ \\
\hline $\begin{array}{l}\text { V534 Tau } \\
M=[1.65-1.69] M_{\odot} \\
v_{\text {rot }}=[14-16] \mu \mathrm{Hz} \\
i=\left[59^{\circ}-79^{\circ}\right]\end{array}$ & $\begin{array}{l}234.2 \\
252.9 \\
307.6 \\
377.9 \\
379.0 \\
448.1 \\
525.0\end{array}$ & $\begin{array}{c}(1,1,1) \\
(1,1,0) \\
(2,0,0),(2,1,1) \\
(2,2-1),(3,0,0) \\
(2,2-1),(3,0,0) \\
(3,1,1) \\
(3,2,-1),(4,0,0) \\
(4,2,-1)\end{array}$ & $\begin{array}{l}\mathrm{HD} 23628 \\
M=[1.82-1.86] M_{\odot} \\
v_{\text {rot }}=[24-26] \mu \mathrm{Hz} \\
i=\left[53^{\circ}-59^{\circ}\right]\end{array}$ & $\begin{array}{l}191.8 \\
201.7 \\
376.6\end{array}$ & $\begin{array}{c}(0,2,2) \\
(1,1,1) \\
(2,2,-2)\end{array}$ \\
\hline $\begin{array}{l}\text { V647 Tau } \\
M=[1.68-1.72] M_{\odot} \\
v_{\text {rot }}=10-11 \mu \mathrm{Hz} \\
i=17^{\circ}-18^{\circ}\end{array}$ & $\begin{array}{l}236.6 \\
304.7 \\
315.6 \\
374.4 \\
405.8 \\
444.1\end{array}$ & $\begin{array}{c}(1,1,1) \\
(1,2,1) \\
(2,0,0),(2,1,1) \\
(2,2,-1) \\
(3,1,0) \\
(3,2,0)\end{array}$ & $\begin{array}{l}\text { HD } 23194 \\
M=[1.78-1.82] M_{\odot} \\
v_{\text {rot }}=[14-23] \mu \mathrm{Hz} \\
i=7^{\circ}-12^{\circ}\end{array}$ & $\begin{array}{l}533.6 \\
574.9\end{array}$ & $\begin{array}{c}(5,1,1),(5,1,-1) \\
(5,2,0),(5,2,1)\end{array}$ \\
\hline
\end{tabular}

V624 Tau with $v_{\text {rot }} \leq 5 \mu \mathrm{Hz}$. HD 23194 and V647 Tau, whose projected rotational velocities are low, $v \sin i=20 \mathrm{~km} \mathrm{~s}^{-1}$, would have equatorial velocities as high as 90 and $70 \mathrm{~km} \mathrm{~s}^{-1}$ respectively.

In the present work the distance is found to be $m_{V}-M_{V}=$ $5.60-5.70$ mag $(132-138 \mathrm{pc})$ and agrees very well with that derived recently by independent determinations using independent techniques and data (Zwahlen et al. 2004; Pan et al. 2004; Munari et al. 2004; Soderblom et al. 2005; Percival et al. 2005). However, although the isochrones computed with subsolar metallicity $(Z=0.015$ and $Y=0.30)$ match well the observational data with a distance modulus of $d=5.39 \mathrm{mag}$ in agreement with that of HIPPARCOS within 1- $\sigma$ error, the resulting solutions (shown by asterisks in Fig. 5) do not lead to good fits. Moreover, the plot of $\epsilon$ against $d$ which we do not reproduce here, shows better fits for further distances. For solar metallicity, in order to reproduce the HIPPARCOS MS of the Pleiades, unrealistically high helium abundances are required (e.g. $Y=0.34$ Belikov et al. 1998, $Y=0.37$ Pinsonneault et al. 1998).

\section{Conclusions}

In this study we have performed a seismological analysis of six $\delta$ Scuti stars belonging to the Pleiades cluster to identify their frequency modes. To the best of our knowledge this group of variables constitutes the most statistically significant sample of $\delta$ Scuti stars analysed in an open cluster to date.

Rotational effects were considered in different stages of the analysis: first when determining the star positions in the HR diagram and second when computing stellar models that approximately reproduce the evolutionary stage of the stars, and finally when computing theoretical oscillation frequencies in order to construct seismic models for target stars. The comparison between observational and computed frequencies was carried out by a least-squares fit. There is a large number of possible solutions partly because neither the equatorial velocity nor the inclination angle are known a priori. In order to limit the number of possible solutions we used the relationship between the amplitude visibility, $S_{l m}$ and the aspect angle, $i$. As a result we found few solutions for each star, suggesting the existence of only $p$ modes of low degree, low radial order in all the stars. For the less massive stars, solutions with at most two radial modes were also possible. These solutions have associated ranges of stellar parameters for each star. Most of the stars could be rapid rotators according to the estimated angle of inclination, $i$.

The best fits between observational and theoretical frequencies are achieved for global cluster parameters of $[\mathrm{Fe} / \mathrm{H}]=$ $0.0668\left(Z_{0}=0.02, X_{0}=0.70\right), A=70-100 \mathrm{Myr}$ and $d=5.60-5.70 \mathrm{mag}$. The derived distance modulus for the Pleiades cluster agrees with that of the main sequence fitting 

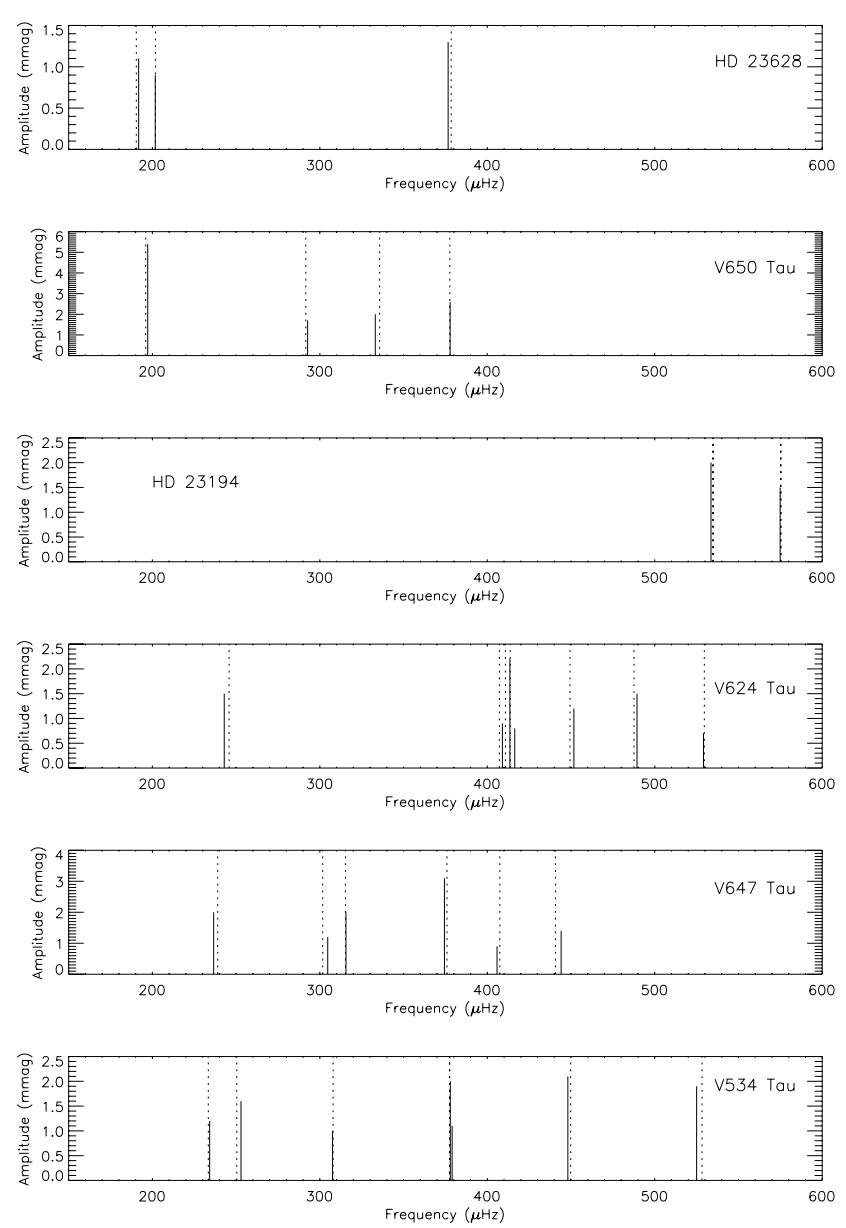

Fig. 7. Comparison of observed (solid lines) and theoretical (dot lines) frequencies of the best fit solutions in C1-models (Table 5).

method, in spite of the fact that the isochrones with sub-solar metallicity closely matches the Pleiades HR diagram with the HIPPARCOS distance.

Acknowledgements. This work has been partially funded by grants AYA2001-1571, ESP2001-4529-PE and ESP2004-03855-C03-03 of the Spanish national research plan. L.F.M acknowledges the partial financial support by the Spanish AECI. J.C.S. acknowledges the financial support by the Spanish Plan of Astronomy and Astrophysics, under project AYA2003-04651, by the Spanish "Consejería de Innovación, Ciencia y Empresa" from the "Junta de Andalucía local government, and by the Spanish Plan Nacional del Espacio under project ESP2004-03855-C03-01.

\section{References}

Aerts, C. 1996, A\&A, 314, 115

Baglin, A, Auvegne M., \& Catala C., and the COROT Team 2001 in Proc. of the SOHO 10/GONG 2000 Workshop: Helio- and Asteroseismology at the Dawn of the Millenium, ESA-SP, 464, 395

Belikov, A. N., Hirte, S., Meusinger, H., et al. 1998, A\&A, 332, 575

Belmonte, J. A., \& Michel, E. 1991, Delta Scuti Newsletter, 3, 16

Boesgaard, A. M., \& Friel, E. D. 1990, ApJ, 351, 457

Bradley, P. A., \& Guzik, J. A. 1996, in Sounding solar and stellar interiors, ed. J. Provost, F. X. Schmider, IAU Symp., 181, 229
Breger, M. 1972, ApJ, 176, 367

Breger, M. 1986, ApJ, 309, 311

Eggen, O. 1986, PASP, 98, 755

Cauglan, G. R., \& Fowler, W. A 1988, Atomic Data and Nuclear Data Tables, 40, 284

Cayrel de Strobel, G. 1990, Mem. Soc. Astron. Ital., 61, 613

Christensen-Dalsgaard, J. 2000, in Delta Scuti \& Related stars, ed. M. Breger, \& H. Montgomery, ASP Conf. Ser., 210, 187

Eggleton, P., Faulkner, J., \& Flannery, B. P. 1973, A\&A, 23, 325

Fox-Machado, L., Álvarez, M., Michel, E., et al. 2002, A\&A, 382, 556

Garrido, R., García-Lobo, E., \& Rodríguez, E. 1990, A\&A, 234, 262

Grevesse, N., \& Noels, A. 1993, in Origin and Evolution of the Elements, ed. N. Prantzos, E. Lagioni-flam, \& M. Casse (Cambridge Univ. Press)

Goupil, M. J., Michel, E., Lebreton, Y., \& Baglin, A. 1993, A\&A, 268, 546

Grenon, M. 2001, in The 11th Cool Stars, Stellar Systems and the Sun, ed. R. J. García López, R. Rebolo, \& M. R. Zapatero Osorio, ASP Conf. Ser., 223, 359

Guzik, J. A., \& Bradley, P. A. 1995, Baltic Astron., 4, 442

Hansen-Ruiz, C. S., \& van Leeuwen, F. 1997, in Hipparcos Venice '97, ESA SP-402, 643

Hernández, M. M., Pérez Hernández, F., Michel, E., et al. 1998, A\&A, 338,511

Iglesias, C. A, \& Rogers, F. J. 1996, ApJ, 464, 943

Kenelly, E. J., \& Walker, G. A. H. 1996, PASP, 108, 327

Kjeldsen, H. 2001, in Proc. of SOHO 10/GONG 2000 workshop Helio- and Asteroseismology at the Dawn of the Millenium, ESA SP-464, 361

Koen, C., Van Rooyen, R., Van Wyk, F., \& Marang, F. 1999, MNRAS, 309,1051

Kurucz, D. W. 1991, in Stellar Atmospheres: Beyond Classical Models, ed. L. Crivellari, I. Hurbeny, \& D. G. Hummer, NATO ASI Series (Dordrecht: Kluwer)

Li, Z. P., Michel, E., Fox Machado, L., et al. 2002, A\&A, 395, 873

Li, Z. P., Michel, E., Fox Machado, L., et al. 2004, A\&A, 420, 283

Liu, Y. Y., Michel, E., Hernández, M. M., et al. 1999, Chinese A\&A, 23, 349

Mermilliod, J.-C. 2000, in Stellar Clusters and Associations, ASP Conf. Ser., 198, 105

Mermilliod, J.-C., Turon, C., Robichon, N., et al. 1997, in Hipparcos Venice '97, ESA SP-402, 643

Michel, E., Goupil, M. J., \& Lebreton, Y. 1991, in Inside the stars, ed. W. Weiss, \& A. Baglin, ASP Conf. Ser., 40, 547

Michel, E., Hernández, M. M., Haudek, G., et al. 1999, A\&A, 342, 253

Michel, E., Chevreton, M., Belmonte, J. A., et al. 2000, in The Impact of Large- Scale Surveys on Pulsating Star Research, ed. L. Szabados, \& D. W. Kurtz, ASP Conf. Ser., 203, 483

Mihalas, D. 1978, Stellar atmospheres, 2nd edn. (Freeman and Cie)

Morel, P. 1997, A\&AS, 124, 597

Morse, J. A., Mathieu, R. D., \& Levine, S. E. 1991, AJ, 101, 1495

Munari, U., Dallaporta, S., Siviero, A., et al. 2004, A\&A, 418, L33

Pan, X., Shao, M., \& Kulkarni, S. 2004, Nature, 427, 326

Pamyatnykh, A. A., Dziembowski, W. A., Handler, G., et al. 1998, A\&A, 333, 141

Percival, S. M., Salaris, M., \& Groenewegen, M. A. 2005, A\&A, 429, 887

Pérez Hernández, F., Claret, A., \& Belmonte, J. A. 1995, A\&A, 295, 113

Pérez Hernández, F., Claret, A., Hernández, M. M., \& Michel, E. 1999, A\&A, 346, 586

Pesnell, W. D. 1985, ApJ, 232, 238 
Pinsonneault, M. H., Stauffer, J. R., \& Hanson, R. B. 1998, ApJ, 504, 170

Rodríguez, E., López-González, M. J., \& López de Coca, P. 2000, A\&AS, 144, 469

Schaller, G., Schaerer, D., Meynet, G., \& Maeder, A. 1992, A\&AS, 96, 269

Schmidt-Kaler, Th. 1982, in Landolt-Börnstein New Series VI/2b (Springer-Verlag)

Scuflaire, R. 1974, A\&A, 36, 107

Sekiguchi, M., \& Fukugita, M. 2000, AJ, 120, 1072

Soderblom, D. R., Nelan, E., Fritz Benedict, G., et al. 2005, AJ, 129, 1616

Soufi, F., Goupil, M. J., \& Dziembowski, W. A. 1998, A\&A, 334, 911

Stello, D., \& Nissen, E. 2001, A\&A, 374, 105

Suárez, J. C. 2002, Ph.D., Sismologie d'étoiles en rotation. Application aux étoiles $\delta$ Scuti, Université Paris (Denis Diderot)
Suárez, J.-C., Michel, E., Haudek, G., et al. 2002, in Radial and Non radial Pulsations as Probes of Stellar Physics, ed. C. Aerts, T. R. Bedding, \& J. Christensen-Dalsgaard, ASP Conf. Ser., 259, 490 Suárez, J. C., Bruntt, H., \& Buzasi, D. 2005, A\&A, 438, 633

Tassoul, J.-L. 1978, in Theory of Rotating Stars (Princeton University Press)

Tranh Minh, F., Léon, L., Goupil, M. J, \& Soufi, F. 1996, in Sounding solar and stellar interiors, ed. J. Provost, F. X. Schmider, IAU Symp., 181, Poster Volume 4-18

Uesugi, A., \& Fukuda, I. 1982, Revised Cat. of Stellar Rotational Velocities, Kyoto University

van Leeuwen, F. 1999, in Harmonizing cosmic distances and scales in a post Hipparcos Era, ed. Egret, \& Heck, ASP Conf. Ser., 176, 52

Viskum, M., Kjeldsen H., Bedding, T. R., et al. 1998, A\&A, 335, 549

Watson, R. D. 1988, Ap\&SS, 140, 255

Zwahlen, N., North, P., Debernardi, Y., et al. 2004, A\&A, 425, L45 